\title{
Synthesis, structure and antimicrobial evaluation of new derivatives of theophylline sugar hydrazones
}

\author{
Mosselhi Abdelnabi Mosselhi, ${ }^{\text {a* }}$ Magda Ahmed Abdallah, ${ }^{b}$ Nadia Hanavy Metwally, \\ Ibrahim Adel El-Desoky, ${ }^{b}$ and Laila Mohamed Break ${ }^{a}$ \\ ${ }^{a}$ Taif University, Faculty of Science, Department of Chemistry, Taif- P.O. 888, Saudi Arabia \\ ${ }^{b}$ Department of Chemistry, Faculty of Science, Cairo University, Giza, 12613 Egypt \\ E-mail: mosselhi2008@,hotmail.com
}

\begin{abstract}
Condensation of 7-ethyl-8-hydrazinotheophylline $\mathbf{1}$ with aldohexoses 2-4 and aldopentoses 5,6 by heating in an aqueous ethanolic solution and in the presence of a catalytic amount of acetic acid gives the corresponding hitherto unknown aldehydo-sugar (7-ethyl-8theophyllinyl)hydrazones 7-11. The structures of the sugar hydrazones 7-11 were confirmed by their elemental analyses and spectral (IR, ${ }^{1} \mathrm{H}$ NMR and FAB-MS) data. Acetylation of the sugar hydrazones 7-11 with acetic anhydride in pyridine at room temperature gave the corresponding poly-O-acetyl derivatives 12-16. Attempted dehydrogenative cyclization of the products 7-11 and 12-16 using various conditions did not afford the corresponding acyclo $C$-nucleosides $(\mathrm{R}=$ sugar or $\mathrm{R}^{`}=$ acetylated sugar) 18. On the other hand, the reaction of 7-11 or 12-16 with bromine in acetic acid and sodium acetate afforded 8-bromo-7-ethyltheophylline 17. The structural elucidation of products is reported and also some of the products were screened for their antimicrobial activity.
\end{abstract}

Keywords: Theophylline, aldohexoses, aldopentoses, condensation, sugar hydrazones

\section{Introduction}

Theophylline derivatives in 7- and 8-positions have been investigated in the respect of their bronchospasmolytic $^{1-4}$ anticancer $^{5}$ and circulatory blood system activity. ${ }^{6}$ A large amount of work has been performed on the fused systems derived from theophylline, including synthetic procedures and structure determination ${ }^{7-15}$ but few of the synthesized heterocyclic derivatives were pharmacologically tested to be anti-inflammatory, ${ }^{16}$ anti P-388-leukemia ${ }^{17}$ and vascular relaxing agents. ${ }^{18}$

Also, acyclo C-nucleosides represent an important class of compounds characterized by an acyclic sugar moiety attached to the heterocycle through a carbon-carbon bond. 
Acyclonucleosides have been recently classified according to the type of glycon rather than the aglycon part. They differ from parent ribonucleosides only by the absence of the ring structure of the pentosyl residue due to the absence of one or more of the bonds of the pentose moiety to give an open-chain residue. ${ }^{19-23}$ They have been reported to have valuable biological activities. ${ }^{19-23}$ Sugar hydrazones of theophyllin-7-ylacetic acid were reported by Klosa ${ }^{24 a}$ in 1958 and some of other reported sugar hydrazones have been found to possess antitumor, antibacterial and antiviral activities. $^{24 \mathrm{~b}}$

In view of the biological importance of theophylline derivatives, sugar hydrazones and acyclonucleosides and in continuation of our studies on the synthesis of purines and nucleoside derivatives as potential bioactive compounds, ${ }^{25-27}$ it was considered worthwhile to study the reaction of 7-ethyl-8-hydrazinotheophylline with sugars. Our goal was the synthesis of $[1,2,4]$ triazolo[4,3-e]theophylline derivatives as new acyclo C-nucleosides via theophylline sugar hydrazones and to explore the biological activities of the products. We report here the synthesis, structure and antimicrobial activity of new theophylline sugar hydrazones.

\section{Results and Discussion}

The required starting 7-ethyl-8-hydrazinotheophylline 1 has been reported. ${ }^{28}$ It was prepared by treatment of 8-chloro-7-ethyltheophylline ${ }^{29}$ with hydrazine hydrate in boiling ethanol. Condensation of equimolar amounts of 7-ethyl-8-hydrazinotheophylline 1 with aldohexoses 2-4 such as, D-glucose 2, D-galactose 3; D-mannose 4, and aldopentoses 5, 6, such as D-ribose 5, Darabinose 6 by heating for 2-6 h in an aqueous ethanolic solution and in the presence of a catalytic amount of acetic acid gave the corresponding aldehydo-sugar (7-ethyl-8theophyllinyl)hydrazones 7-11 (Scheme 1).

According to our knowledge, none of these hydrazones 7-11 has been reported hitherto. The structures of obtained hydrazones 7-11 were confirmed by their elemental analyses and spectral (IR, ${ }^{1} \mathrm{H}$ NMR and FAB-MS) data (see Experimental Section). For example, their ${ }^{1} \mathrm{H}$ NMR spectra in DMSO- $d_{6}$ revealed in each case, a characteristic signal in the region $\delta=7.43-7.54$ assignable to the azamethine $\mathrm{N}=\mathrm{CH}$ proton. Also, ${ }^{1} \mathrm{H}$ NMR spectra revealed methyl groups at $\delta=$ $3.17,3.31$ and an ethyl group signals at $\delta=1.20\left(\mathrm{CH}_{3}\right)$ and $4.2\left(\mathrm{CH}_{2}\right)$. The alditolyl group protons ( $\mathrm{CHOH}$ proton signals of the sugar chain) were associated with the solvent absorption (DMSO- $d_{6}$ ) forming a broad signal at $\delta=3.20-4.06$ ( $\mathrm{CH}$ protons) and 4.20-5.79 (OH protons). The IR spectra of sugar hydrazones showed bands at $3243-3462 \mathrm{~cm}^{-1}$ due to the $\mathrm{OH}$ groups, bands at $1629-1699 \mathrm{~cm}^{-1}$ due to the $\mathrm{C}=\mathrm{O}$ groups. The mass spectra of the hydrazones 9-11 by using $\mathrm{FAB}$ technique revealed $\mathrm{M}^{+}$and $\mathrm{M}+1^{+}$peaks with intensities of $20-47 \%$, while that of hydrazones 7 and 8 appeared with intensities of 46 and $76 \%$, respectively. 


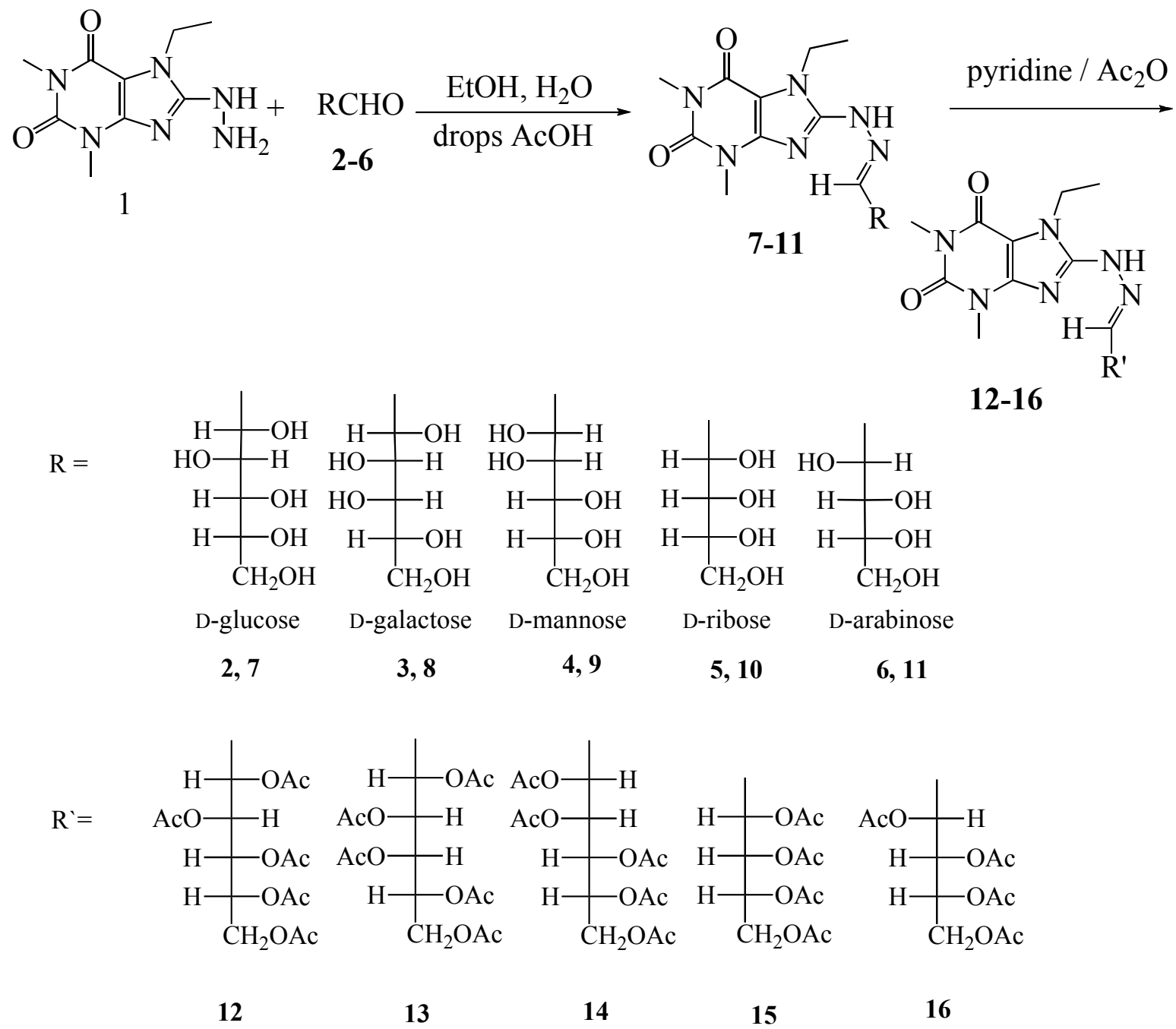

Scheme 1. Synthesis of hydrazones 7-11 and acetylated hydrazones 12-16.

Acetylation of the sugar hydrazones 7-11 with acetic anhydride in pyridine at room temperature gave the corresponding poly-O-acetyl derivatives 12-16 (Scheme 1). The IR absorptions of the acetylated sugar hydrazones 12-16 formed showed absorption bands in the carbonyl frequency region at $1745-1752 \mathrm{~cm}^{-1}$ indicating the introduction of O-acetyl groups. Their ${ }^{1} \mathrm{H}$ NMR spectra exhibited signals in the region of $\delta=1.85-2.16$ confirming the presence of OAc methyl protons. FAB-Mass spectra of 12-16 showed characteristic $\mathrm{M}+1^{+}$peaks with intensity of $35-52 \%$.

Attempted dehydrogenative cyclization of sugar hydrazones 7-11 or their acetylated derivatives 12-16 with ferric chloride in ethanol, ${ }^{30,31}$ copper dichloride in dimethylformamide, ${ }^{32}$ bromine in water ${ }^{33}$ or in acetic acid, ${ }^{34}$ bromine in acetic acid/sodium acetate, ${ }^{24}$ bromine in acetic acid, sodium acetate and acetic anhydride ${ }^{35}$ or thionyl chloride, ${ }^{36}$ as previously used for the cyclization of related sugar hydrazones, did not proceed and no crystalline derivatives of the acyclo $C$-nucleosides $\left(\mathrm{R}=\right.$ sugar or $\mathrm{R}^{`}=$ acetylated sugar) 18 (Scheme 2$)$ could be isolated. The 
reaction of 7-11 or 12-16 with bromine in acetic acid and sodium acetate did not afford the respective cyclized product 1,2,4-triazolo[4,3-e]theophylline 18, but instead 8-bromo-7ethyltheophylline $17^{37}$ identified by its elemental and spectral analysis (Scheme 2). Further evidence for structure $\mathbf{1 7}$ was based on its reaction with hydrazine hydrate giving the starting compound $\mathbf{1} .^{28}$

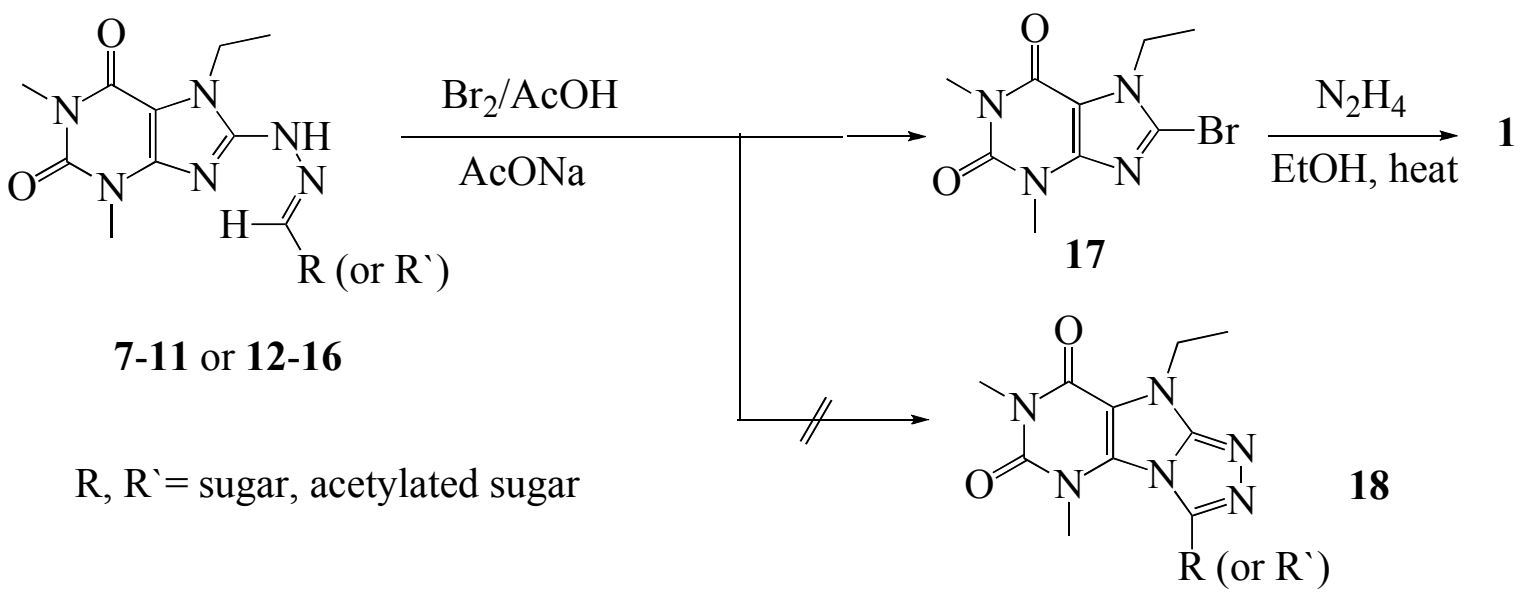

\section{Scheme 2}

Doering ${ }^{32}$ reported that heterocyclization of butanal caffein-8-ylhydrazone 19 was achieved by using copper dichloride to give 5,7,9-trimethyl-3-propyl-[1,2,4]triazolo[4,3-e]purine-6,8dione 20 (Scheme 3) in poor yield (15\%). He also suggested that the lower selectivity in the oxidation of 19 indicated that a five-membered ring is less favorable and the difficulties of the heterocyclization of $\mathbf{1 9}$ could also be caused by the sterically demanding effect of the methyl group at position 5. This result seems to be in accord with our findings that electrocyclization of sugar hydrazones 7-11 or acetylated sugar hydrazones 12-16 is difficult though this is still under investigation.<smiles>CCCC(C)NNc1nc2c3cn[nH]c3n(C)c2c(=O)n(C)c1=O</smiles><smiles>CC(C)(C)C(C)(C)C(C)(C)C</smiles><smiles>CCCc1nnc2n1C1C(C(=O)N(C)C(=O)N1C)N2C</smiles>

\section{Scheme 3}




\section{Antimicrobial activity}

The compounds 9 and 12-16 were tested for their antimicrobial activities using four fungal species, namely Aspergillus fumigatus AF, Penicillium italicum PI, Syncephalastrum racemosum SR and Candida albicans $\mathbf{C A}$, as well as four bacterial species namely Escherichia coli EC, Bacillus subtilis BS, Pseudomonas aeruginosa PA and Staphylococcus aureus $\mathbf{S A}$. The microorganisms were tested against the activity of solutions of concentrations of 5.0, 2.5 and 1.0 $\mathrm{mg} / \mathrm{ml}$ of each compound and using an inhibition zone diameter in $\mathrm{cm}$ (IZD) as criterion for the antimicrobial activity. The fungicide Terbinafin and the bactericide Chloramphenicol were used as references to evaluate the potency of the tested compounds under the same conditions. The results are depicted in Table 1. Compound $\mathbf{9}$ showed the highest degree of inhibition against AF, PI and SA and weak inhibition against SR. Compounds 9, 12, 13, 15 and 16 displayed a considerable degree of inhibition against SR and EC. While $\mathbf{1 2}$ had a considerable degree of inhibition against BS, compounds 13, 15 and 16 had only weak inhibition against BS. Moreover, compounds 9, 12, 15 and $\mathbf{1 6}$ have weak inhibition against PI. All compounds, except 9 and 14, exhibited no activity against AF, SA and PA. All compounds except 12 showed no activity against CA.

Table 1. Antimicrobial activity

\begin{tabular}{|c|c|c|c|c|c|c|c|c|c|c|c|c|c|c|c|c|c|c|c|c|c|}
\hline $\begin{array}{l}\text { Comp. } \\
\text { No }\end{array}$ & & 9 & & & 12 & & & 13 & & & 14 & & & 15 & & & 16 & & & St. & \\
\hline $\begin{array}{l}\text { Conc. } \\
\mathrm{mg} / \mathrm{ml}\end{array}$ & 5 & 2.5 & 1 & 5 & 2.5 & 1 & 5 & 2.5 & 1 & 5 & 2.5 & 1 & 5 & 2.5 & 1 & 5 & 2.5 & 1 & 5 & 2.5 & 1 \\
\hline $\mathrm{AF}$ & + & + & - & - & - & - & - & - & - & ++ & + & - & - & - & - & - & - & - & +++ & +++ & ++ \\
\hline PI & + & + & - & + & - & - & - & - & - & ++ & ++ & ++ & + & + & - & + & + & - & +++ & +++ & ++ \\
\hline SR & ++ & + & - & + & + & - & + & + & - & + & + & + & + & + & - & + & - & - & +++ & +++ & +++ \\
\hline CA & - & - & - & + & + & - & - & - & - & - & - & - & - & - & - & - & - & - & ++ & ++ & ++ \\
\hline SA & + & - & - & - & - & - & - & - & - & ++ & + & - & - & - & - & - & - & - & ++ & ++ & ++ \\
\hline PA & + & - & - & - & - & - & - & - & - & + & - & - & - & - & - & - & - & - & +++ & +++ & ++ \\
\hline BS & - & - & - & ++ & + & - & + & + & - & - & - & - & + & + & - & + & - & - & +++ & +++ & ++ \\
\hline $\mathrm{EC}$ & + & - & - & + & + & + & + & + & + & + & - & - & + & + & + & + & + & + & ++ & ++ & ++ \\
\hline
\end{tabular}

St. - reference standard; Chloramphenicol (as a standard antifungal); Terbinafin (as a standard antibacterial. The test was done using diffusion agar technique. Well diameter: $0.6 \mathrm{~cm} . . .(100 \mu \mathrm{l}$ of each conc. was tested). Inhibiton values $=0.1-1.5 \mathrm{~cm}$ (beyond control $=+$ ), 0.6-1.0 (beyond control $=++), 1.1-1.5 \mathrm{~cm}$ (beyond control $=+++), 0$ (not detected $=-$ ). 


\section{Conclusions}

In conclusion we have synthesized some new antimicrobially active derivatives of theophylline sugar hydrazones 7-11 and their acetylation products 12-16. Attempted dehydrogenative cyclization of the sugar hydrazones failed. Further investigations are currently underway aimed at the synthesis of biological interesting $C$-nucleosides.

\section{Experimental Section}

General. All evaporations were carried out under reduced pressure at $60^{\circ} \mathrm{C}$. TLC was carried out on aluminum sheet silica gel 60 (Fluka) and detected by short wavelength UV light. All melting points were measured on an electrothermal melting point apparatus and are uncorrected. The ${ }^{1} \mathrm{H}$ NMR spectra were recorded in deuterated chloroform $\left(\mathrm{CDCl}_{3}\right)$ or dimethyl sulfoxide (DMSO- $d_{6}$ ) at $300 \mathrm{MHz}$ on a Varian Mercury VXR-300 NMR spectrometer and ${ }^{13} \mathrm{C}$ NMR spectra were recorded in dimethyl sulfoxide (DMSO- $d_{6}$ ) at $75 \mathrm{MHz}$ using a Jeol-300 NMR spectrometer. Chemical shifts were related to that of the solvent. The infrared spectra were recorded in potassium bromide discs on a Pye-Unicam, SP300 and Shimadzu, FT IR 8101 PC infrared spectrophotometers. Biological activity was assessed at the Regional Center for Mycology and Biotechnology at Al-azhar University, Cairo, Egypt. FAB mass spectrometry was carried out at the Institute of Organic Chemistry, Hamburg University, Germany. Elemental analyses were carried out at the Microanalytical Center of Cairo University, Giza, Egypt. Theophylline, sugars, bromine, ethyl iodide and hydrazine hydrate were purchased from Aldrich Chemical Co. Ethanol, methanol, diethyl ether, petroleum ether, chloroform, $N, N$ dimethylformamide, nitric acid, hydrochloric acid, sodium acetate trihydrate, potassium carbonate, pyridine, glacial acetic acid and acetic anhydride were purchased from EL-Nasr Pharmaceutical and Chemical Co. (ADWIC), Egypt.

7-Ethyl-8-hydrazinotheophylline $\mathbf{1},{ }^{28}$ 8-chloro-7-ethyltheophylline, ${ }^{29} 8$-nitrotheophylline, ${ }^{38}$ and 8 -chlorotheophylline ${ }^{38}$ were prepared according to the in literature reported methods.

\section{General procedure for synthesis of sugar 7-ethyl-8-theophyllinyl-hydrazones 7-11}

To a suspension of 7-ethyl-8-hydrazinotheophylline 1 ( $2.38 \mathrm{~g}, 10 \mathrm{mmol})$ in ethanol $(30 \mathrm{ml})$, was added a solution of the appropriate sugar 2-6 $(10 \mathrm{mmol})$ in water $(10 \mathrm{ml})$ and few drops of glacial acetic acid. The mixture was heated at reflux until reaction was judged complete by TLC (2-6 h). The solid product formed upon cooling was filtered off, washed with the minimum amount of ethanol, dried and finally recrystallized from ethanol to afford the respective hydrazones 7-11. The physical constants and the spectral data of the products 7-11 are listed below:

D-Glucose-7-ethyl-8-theophyllinylhydrazone 7. Yield 70\%; white crystals, mp $195{ }^{\circ} \mathrm{C}$; IR $(\mathrm{KBr})$ v $3446(\mathrm{OH}), 3248(\mathrm{NH}), 1697,1655(\mathrm{C}=\mathrm{O}) \mathrm{cm}^{-1} ;{ }^{1} \mathrm{H}$ NMR (300 MHz, DMSO-d 6 ) $\delta 1.25$ 
(t, $\left.3 \mathrm{H}, \mathrm{N}-\mathrm{CH}_{2} \mathrm{CH}_{3}\right), 3.02(\mathrm{~m}, 2 \mathrm{H}$, alditolyl $2 \mathrm{H}), 3.17-3.48\left(\mathrm{~m}, 8 \mathrm{H}\right.$, alditolyl $\left.2 \mathrm{H}, 2 \mathrm{~N}-\mathrm{CH}_{3}\right), 3.65-$ $3.89(2 \mathrm{~m}, 2 \mathrm{H}$, alditolyl $2 \mathrm{H}), 4.22-4.50\left(\mathrm{~m}, 4 \mathrm{H}, \mathrm{N}-\mathrm{CH}_{2} \mathrm{CH}_{3}+2 \mathrm{OH}\right.$, exchangeable), $4.89(\mathrm{t}, 1 \mathrm{H}$, $\mathrm{OH}$, exchangeable), 5.22, 5.79 (2d, 2H, 2OH, exchangeable), $7.43(\mathrm{~d}, 1 \mathrm{H}, \mathrm{N}=\mathrm{CH}), 8.41(\mathrm{~s}, 1 \mathrm{H}$, exchangeable, =N-NH-); ${ }^{1} \mathrm{C}$ NMR $\left(75 \mathrm{MHz}, \mathrm{DMSO}-\mathrm{d}_{6}\right) \delta 14.7\left(\mathrm{C}-7 \mathrm{CH}_{3}\right), 26.9\left(\mathrm{C}-7 \mathrm{CH}_{2}\right), 28.9$ (C-3), 38.8 (C-1), 61.2-77.5 (C-alditol), 101.5 (C-5), 144.5 (C-4), $147.4(\mathrm{C}-8), 150.6(\mathrm{~N}=\mathrm{CH})$, 152.5 (C-2), 153.9 (C-6); MS: m/z (\%) = $401\left(\mathrm{M}+1^{+}, 76\right), 400\left(\mathrm{M}^{+}, 46\right), 307$ (63), 289 (40), 279 (14), 267 (8.0), 238 (16), 222 (100), 219 (8.0), 216 (5.0). Anal. calcd. for $\mathrm{C}_{15} \mathrm{H}_{24} \mathrm{~N}_{6} \mathrm{O}_{7}$ (400): C, 45.00; H, 6.04; N, 20.99. Found: C, 45.00; H, 6.00; N, 21.00\%.

D-Galactose-7-ethyl-8-theophyllinylhydrazone 8. Yield 73\%; white crystals, mp 213-214 ${ }^{\circ} \mathrm{C}$; IR $(\mathrm{KBr})$ v $3462(\mathrm{OH}), 3235(\mathrm{NH}), 1696,1629(\mathrm{C}=\mathrm{O}) \mathrm{cm}^{-1} ;{ }^{1} \mathrm{H}$ NMR $\left(300 \mathrm{MHz}, \mathrm{DMSO}-d_{6}\right) \delta$ $1.20\left(\mathrm{t}, 3 \mathrm{H}, \mathrm{N}-\mathrm{CH}_{2} \mathrm{CH}_{3}\right), 3.18\left(\mathrm{~s}, 3 \mathrm{H}, \mathrm{N}-\mathrm{CH}_{3}\right), 3.24-3.41\left(\mathrm{~m}, 5 \mathrm{H}\right.$, alditolyl $\left.2 \mathrm{H}, \mathrm{N}-\mathrm{CH}_{3}\right), 3.53$, $3.83(2 \mathrm{~m} ., 4 \mathrm{H}$, alditolyl $4 \mathrm{H}), 4.23-4.28\left(\mathrm{~m}, 3 \mathrm{H}, \mathrm{N}-\mathrm{CH}_{2} \mathrm{CH}_{3}+\mathrm{OH}\right.$, exchangeable), 4.47 (t, $1 \mathrm{H}$, $\mathrm{OH}$, exchangeable), 4.69, 5.21, $5.74(3 \mathrm{~d}, 3 \mathrm{H}, 3 \mathrm{OH}$, exchangeable), $7.54(\mathrm{~d}, 1 \mathrm{H}, \mathrm{N}=\mathrm{CH}), 8.46(\mathrm{~s}$, $1 \mathrm{H}$, exchangeable, $=\mathrm{N}-\mathrm{NH}-)$; ${ }^{3} \mathrm{C} \mathrm{NMR}\left(75 \mathrm{MHz}, \mathrm{DMSO}-d_{6}\right) \delta 15.0\left(\mathrm{C}-7 \mathrm{CH}_{3}\right), 27.3\left(\mathrm{C}-7 \mathrm{CH}_{2}\right)$, 29.2 (C-3), 39.1 (C-1), 60.7-76.6 (C-alditol), 101.7 (C-5), 143.5 (C-4), 150.9 (C-8), 152.8 $(\mathrm{N}=\mathrm{CH}), 152.9(\mathrm{C}-2), 154.5(\mathrm{C}-6) ; \mathrm{MS}: m / z(\%)=401\left(\mathrm{M}+1^{+}, 77\right), 400\left(\mathrm{M}^{+}, 47\right), 391(8), 308$ (26), 307 (100), 289 (54), 279 (10), 267 (8.0), 238 (17), 223 (39), 222 (84). Anal. calcd. for $\mathrm{C}_{15} \mathrm{H}_{24} \mathrm{~N}_{6} \mathrm{O}_{7}$ (400): C, 45.00; H, 6.04; N, 20.99. Found: C, 44.98; H, 6.01; N, 20.95\%.

D-Mannose-7-ethyl-8-theophyllinylhydrazone 9. Yield 78\%; yellowish white crystals, mp 180 ${ }^{\circ} \mathrm{C}$; IR (KBr) v $3407(\mathrm{OH}), 3245(\mathrm{NH}), 1684,1657(\mathrm{C}=\mathrm{O}) \mathrm{cm}^{-1}$; ${ }^{1} \mathrm{H}$ NMR (300 MHz, DMSO-d 6 ) $\delta 1.26\left(\mathrm{t}, 3 \mathrm{H}, \mathrm{N}-\mathrm{CH}_{2} \mathrm{CH}_{3}\right), 3.19-3.71(\mathrm{~m}, 11 \mathrm{H}$, alditolyl 5H, 2N-CH$), 4.05(\mathrm{~m}, 1 \mathrm{H}$, alditolyl $\mathrm{H})$, 4.23-4.34 (m, 5H, N-CH $\mathrm{CH}_{3}+3 \mathrm{OH}$, exchangeable), $4.43(\mathrm{t}, 1 \mathrm{H}, \mathrm{OH}$, exchangeable), 5.16 (d, $1 \mathrm{H}, \mathrm{OH}$, exchangeable), $7.43(\mathrm{~d}, 1 \mathrm{H}, \mathrm{N}=\mathrm{CH}), 10.92(\mathrm{~s}, 1 \mathrm{H}$, exchangeable, $=\mathrm{N}-\mathrm{NH}-)$; ${ }^{3} \mathrm{C}$ NMR $\left(75 \mathrm{MHz}, \mathrm{DMSO}-\mathrm{d}_{6}\right) \delta 15.2\left(\mathrm{C}-7 \mathrm{CH}_{3}\right), 26.9\left(\mathrm{C}-7 \mathrm{CH}_{2}\right), 28.9(\mathrm{C}-3), 38.8(\mathrm{C}-1), 63.4-70.8(\mathrm{C}-$ alditol), 101.7 (C-5), 146.0 (C-4), 148.7 (C-8), $149.6(\mathrm{~N}=\mathrm{CH}), 150.6$ (C-2), 152.6 (C-6); MS: $\mathrm{m} / \mathrm{z}(\%)=401\left(\mathrm{M}^{+}+1,30\right), 400\left(\mathrm{M}^{+}, 19\right), 308$ (22), 307 (100), 289 (45), 273 (7.0), 224 (7.0), 222 (42), Anal. calcd. for $\mathrm{C}_{15} \mathrm{H}_{24} \mathrm{~N}_{6} \mathrm{O}_{7}$ (400): C, 45.00; H, 6.04; N, 20.99. Found: C, 45.05; H, 5.99; N, 20.93\%.

D-Ribose-7-ethyl-8-theophyllinylhydrazone 10. Yield 55\%; white crystals, mp $205{ }^{\circ} \mathrm{C}$; IR $(\mathrm{KBr})$ v $3356(\mathrm{OH}), 3235(\mathrm{NH}), 1699,1638(\mathrm{C}=\mathrm{O}) \mathrm{cm}^{-1} ;{ }^{1} \mathrm{H}$ NMR (300 MHz, DMSO-d 6 ) $\delta 1.25$ $\left(\mathrm{t}, 3 \mathrm{H}, \mathrm{N}-\mathrm{CH}_{2} \mathrm{CH}_{3}\right), 3.19\left(\mathrm{~s}, 3 \mathrm{H}, \mathrm{N}-\mathrm{CH}_{3}\right), 3.31-3.61$ (m, 7H, alditolyl 4H, N-CH $), 4.20-4.34(\mathrm{~m}$, $4 \mathrm{H}$, alditolyl $\mathrm{H}, \mathrm{N}-\mathrm{CH}_{2} \mathrm{CH}_{3}, \mathrm{OH}$, exchangeable), 4.52, 4.85, 5.16 (3d, 3H, 3OH, exchangeable), $7.46(\mathrm{~d}, 1 \mathrm{H}, \mathrm{N}=\mathrm{CH}), 10.90(\mathrm{~s}, 1 \mathrm{H}$, exchangeable, $=\mathrm{N}-\mathrm{NH}-)$; ${ }_{3} \mathrm{C}$ NMR $\left(75 \mathrm{MHz}, \mathrm{DMSO}-\mathrm{d}_{6}\right) \delta$ $15.4\left(\mathrm{C}-7 \mathrm{CH}_{3}\right), 27.2\left(\mathrm{C}-7 \mathrm{CH}_{2}\right), 29.2$ (C-3), 38.8 (C-1), 63.1-74.1 (C-alditol), 101.9 (C-5), 147.8 (C-4), 148.0 (C-8), $149.9(\mathrm{~N}=\mathrm{CH}), 150.9$ (C-2), 152.9 (C-6); MS: m/z (\%) = $371\left(\mathrm{M}+1^{+}, 29\right)$, $370\left(\mathrm{M}^{+}, 15\right), 307$ (100), 289 (50), 273 (9.0), 260 (5.0), 242 (6.0), 222 (26), 215 (5.0). Anal. calcd. for $\mathrm{C}_{14} \mathrm{H}_{22} \mathrm{~N}_{6} \mathrm{O}_{6}(370)$ : C, 45.40; H, 5.99; N, 22.69. Found: C, 45.15; H, 5.94; N, 22.70\% D-Arabinose-7-ethyl-8-theophyllinylhydrazone 11. Yield 55\%; white crystals, mp $205{ }^{\circ} \mathrm{C}$; IR $(\mathrm{KBr})$ v $3356(\mathrm{OH}), 3208(\mathrm{NH}), 1688,1655(\mathrm{C}=\mathrm{O}) \mathrm{cm}^{-1} ;{ }^{1} \mathrm{H}$ NMR (300 MHz, DMSO-d $) \delta 1.25$ (t, $\left.3 \mathrm{H}, \mathrm{N}-\mathrm{CH}_{2} \mathrm{CH}_{3}\right), 3.19\left(\mathrm{~s}, 3 \mathrm{H}, \mathrm{N}-\mathrm{CH}_{3}\right), 3.31-3.58$ (m, 7H, alditolyl 4H, N-CH$), 4.28-4.34$ (m, 
4H, alditolyl $\mathrm{H}, \mathrm{N}-\mathrm{CH}_{2} \mathrm{CH}_{3}, \mathrm{OH}$, exchangeable), 4.57 (m, 2H, 2OH, exchangeable), 4.96 (d, 1H, $\mathrm{OH}$, exchangeable), $7.51(\mathrm{~d}, 1 \mathrm{H}, \mathrm{N}=\mathrm{CH}), 10.91(\mathrm{~s}, 1 \mathrm{H}$, exchangeable, $=\mathrm{N}-\mathrm{NH}-)$; ${ }^{3} \mathrm{C}$ NMR $(75$ $\left.\mathrm{MHz}, \mathrm{DMSO}_{6}\right) \delta 15.3\left(\mathrm{C}-7 \mathrm{CH}_{3}\right), 27.1\left(\mathrm{C}-7 \mathrm{CH}_{2}\right), 29.1(\mathrm{C}-3), 38.7(\mathrm{C}-1), 63.2-73.3(\mathrm{C}-$ alditol), 101.9 (C-5), 147.8 (C-4), 148.8 (C-8), 149.8 (N=CH), 150.8 (C-2), 152.8 (C-6); MS: $\mathrm{m} / \mathrm{z}(\%)=371\left(\mathrm{M}+1^{+}, 38\right), 307(100), 289(54), 257(8.0), 222$ (29). Anal. calcd. for $\mathrm{C}_{14} \mathrm{H}_{22} \mathrm{~N}_{6} \mathrm{O}_{6}$ (370): C, 45.40; H, 5.99; N, 22.69. Found: C, 45.35; H, 5.89; N, 22.65\%.

\section{General procedure for synthesis of peracetylated sugar 7-ethyl-8-theophyllinylhydrazone 12-16}

To a solution of the appropriate sugar 7-ethyl-8-theophyllinylhydrazone 7-11 (4 mmol) in the minimum amount of pyridine $(1-4 \mathrm{ml})$, was added acetic anhydride $(15 \mathrm{ml})$. The mixture was stirred at room temperature until reaction was judged complete by TLC. The mixture was poured into crushed ice and stirred leading to the precipitation of a yellowish-white solid. The precipitate was collected by filtration, washed with water, dried and finally recrystallized from ethanol to afford the respective peracetylated sugar hydrazone 12-16, the physical constants and the spectral data of the products 12-16 are listed below.

2,3,4,5,6-Penta-O-acetyl-D-glucose-7-ethyl-8-theophyllinyl-hydrazone 12 . Yield 55\%; yellow crystals, mp $122{ }^{\circ} \mathrm{C}$; IR (KBr) v $3228(\mathrm{NH}), 1750\left(\mathrm{COCH}_{3}\right), 1699,1655(\mathrm{C}=\mathrm{O}) \mathrm{cm}^{-1}$; ${ }^{1} \mathrm{H}$ NMR $\left(300 \mathrm{MHz}, \mathrm{CDCl}_{3}\right) \delta 1.44\left(\mathrm{t}, 3 \mathrm{H}, \mathrm{N}-\mathrm{CH}_{2} \mathrm{CH}_{3}\right), 2.02-2.16\left(\mathrm{~m}, 15 \mathrm{H}, 5 \mathrm{COCH}_{3}\right), 3.39,3.47(2 \mathrm{~s}$, $\left.6 \mathrm{H}, 2 \mathrm{~N}-\mathrm{CH}_{3}\right), 4.11,4.25,5.11,5.43,5.60\left(5 \mathrm{~m}, 6 \mathrm{H}\right.$, alditolyl H), 4.49 (q, 2H, N-CH $\left.2 \mathrm{CH}_{3}\right), 7.20$ $(\mathrm{d}, 1 \mathrm{H}, \mathrm{N}=\mathrm{CH}), 8.64(\mathrm{~s}, 1 \mathrm{H}$, exchangeable, $=\mathrm{N}-\mathrm{NH}-)$; ${ }^{13} \mathrm{C} \mathrm{NMR}\left(75 \mathrm{MHz}, \mathrm{DMSO}-\mathrm{d}_{6}\right) \delta 15.2(\mathrm{C}-7$ $\left.\mathrm{CH}_{3}\right), 20.1-20.5\left(5 \mathrm{CH}_{3}\right), 27.2\left(\mathrm{C}-7 \mathrm{CH}_{2}\right), 29.2(\mathrm{C}-3), 38.8(\mathrm{C}-1), 61.5-75.0$ (C-alditol), $101.5(\mathrm{C}-$ 8), 144.8 (C-4), 148.6 (C-8), $149.6(\mathrm{~N}=\mathrm{CH}), 150.5$ (C-2), 152.9 (C-6); 169.1-171.5 (5 CO); MS: $\mathrm{m} / \mathrm{z}(\%)=611\left(\mathrm{M}+1^{+}, 30\right), 551(2.0), 508$ (1.0), 431 (1.0), 391 (3.0), 330 (2.0), 291 (3.0), 275 (4.0), 235 (3.0), 224 (26), 222 (100). Anal. calcd. for $\mathrm{C}_{25} \mathrm{H}_{34} \mathrm{~N}_{6} \mathrm{O}_{12}(610): \mathrm{C}, 49.18 ; \mathrm{H}, 5.61$; N, 13.76. Found: C, 49.00; H, 5.65; N, 13.72\%.

$\mathbf{2 , 3 , 4 , 5 , 6 - P e n t a - O} \boldsymbol{O}$-acetyl-D-galactose-7-ethyl-8-theophyllinyl-hydrazone $\quad \mathbf{1 3}$. Yield 78\%; yellow crystals, mp $159-161{ }^{\circ} \mathrm{C}$; IR $(\mathrm{KBr})$ v $3234(\mathrm{NH}), 1750\left(\mathrm{COCH}_{3}\right), 1695,1655(\mathrm{C}=\mathrm{O}) \mathrm{cm}^{-1}$; ${ }^{1} \mathrm{H}$ NMR (300 MHz, DMSO-d 6 ) $\delta 1.41$ (t, 3H, N-CH $\mathrm{CH}_{3}$ ), 1.90-2.10 (m, 15H, $\left.5 \mathrm{COCH}_{3}\right), 3.26$, $3.37\left(2 \mathrm{~s}, 6 \mathrm{H}, 2 \mathrm{~N}-\mathrm{CH}_{3}\right), 4.00-4.25\left(\mathrm{~m}, 4 \mathrm{H}\right.$, alditolyl $\left.2 \mathrm{H}, \mathrm{N}-\mathrm{CH}_{2} \mathrm{CH}_{3}\right), 4.66,5.42,5.72(3 \mathrm{~m}, 4 \mathrm{H}$, alditolyl H), $7.26(\mathrm{~d}, 1 \mathrm{H}, \mathrm{N}=\mathrm{CH}), 11.92\left(\mathrm{~s}, 1 \mathrm{H}\right.$, exchangeable, =N-NH-); ${ }^{\mathrm{C}} \mathrm{NMR}(75 \mathrm{MHz}$, DMSO-d $\left.{ }_{6}\right) \delta 15.5\left(\mathrm{C}-7 \mathrm{CH}_{3}\right), 20.2-21.0\left(5 \mathrm{CH}_{3}\right), 27.4\left(\mathrm{C}-7 \mathrm{CH}_{2}\right), 29.8(\mathrm{C}-3), 38.4(\mathrm{C}-1), 61.0-$ 74.3 (C-alditol), 101.9 (C-5), 146.2 (C-4), 148.0 (C-8), $149.5(\mathrm{~N}=\mathrm{CH}), 150.7$ (C-2), 152.5 (C-6); 169.7-171.7 (5 CO); MS: m/z (\%) = $611\left(\mathrm{M}+1^{+}, 33\right), 307$ (20), 289 (11), 249 (7.0), 224 (30), 222 (100). Anal. calcd. for $\mathrm{C}_{25} \mathrm{H}_{34} \mathrm{~N}_{6} \mathrm{O}_{12}$ (610): C, 49.18; H, 5.61; N, 13.76. Found: C, 49.20; H, $5.56 ; \mathrm{N}, 13.73 \%$.

2,3,4,5,6-Penta- $O$-acetyl-D-mannose-7-ethyl-8-theophyllinylhydrazone $\quad \mathbf{1 4}$. Yield 41\%; yellow crystals, mp $145-147{ }^{\circ} \mathrm{C}$; IR $(\mathrm{KBr})$ v $3235(\mathrm{NH}), 1752\left(\mathrm{COCH}_{3}\right), 1696,1654(\mathrm{C}=\mathrm{O}) \mathrm{cm}^{-1}$; ${ }^{1} \mathrm{H}$ NMR (300 MHz, DMSO-d 6 ) $\delta 1.39$ (t, 3H, N-CH $\left.\mathrm{CH}_{3}\right), 1.89-2.12\left(\mathrm{~m}, 15 \mathrm{H}, 5 \mathrm{COCH}_{3}\right), 3.26$, 
$3.37\left(2 \mathrm{~s}, 6 \mathrm{H}, 2 \mathrm{~N}-\mathrm{CH}_{3}\right), 3.96,4.23,4.64,5.46,5.91\left(5 \mathrm{~m}, 8 \mathrm{H}\right.$, alditolyl $\left.\mathrm{H}, \mathrm{N}-\mathrm{CH}_{2} \mathrm{CH}_{3}\right), 7.20$ (d, $1 \mathrm{H}, \mathrm{N}=\mathrm{CH}), 11.96(\mathrm{~s}, 1 \mathrm{H}$, exchangeable, $=\mathrm{N}-\mathrm{NH}-)$; ${ }^{3} \mathrm{C}$ NMR $\left(75 \mathrm{MHz}, \mathrm{DMSO}-\mathrm{d}_{6}\right) \delta 15.0(\mathrm{C}-7$ $\left.\mathrm{CH}_{3}\right), 20.5-21.1\left(5 \mathrm{CH}_{3}\right), 27.8\left(\mathrm{C}-7 \mathrm{CH}_{2}\right), 29.8(\mathrm{C}-3), 39.0(\mathrm{C}-1), 61.5-72.5$ (C-alditol), 102.0 (C5), $145.5(\mathrm{C}-4), 147.9(\mathrm{C}-8), 149.3(\mathrm{~N}=\mathrm{CH}), 150.6(\mathrm{C}-2), 152.0(\mathrm{C}-6) ; 169.5-172.1$ (5 CO); MS: $\mathrm{m} / \mathrm{z}(\%)=611\left(\mathrm{M}+1^{+}, 34\right), 609$ (17), 551 (2.0), 333 (2.0), 291 (2.0), 275 (4.0), 250 (7.0), 235 (3.0), 224 (7), 222 (100). Anal. calcd. for $\mathrm{C}_{25} \mathrm{H}_{34} \mathrm{~N}_{6} \mathrm{O}_{12}$ (610): C, 49.18; H, 5.61; N, 13.76. Found: C, 49.25; H, 5.60; N, 13.69

2,3,4,5-Tetra- $\boldsymbol{O}$-acetyl-D-ribose-7-ethyl-8-theophyllinylhydrazone 15 . Yield 26\%; yellow crystals, mp 158-159 ${ }^{\circ} \mathrm{C}$; IR (KBr) v $3235(\mathrm{NH}), 1749\left(\mathrm{COCH}_{3}\right), 1696,1660(\mathrm{C}=\mathrm{O}) \mathrm{cm}^{-1} ;{ }^{1} \mathrm{H}$ NMR (300 MHz, DMSO-d $)_{6} \delta 1.38\left(\mathrm{t}, 3 \mathrm{H}, \mathrm{N}-\mathrm{CH}_{2} \mathrm{CH}_{3}\right), 1.85-2.11\left(\mathrm{~m}, 12 \mathrm{H}, 4 \mathrm{COCH}_{3}\right), 3.24$, $3.35\left(2 \mathrm{~s}, 6 \mathrm{H}, 2 \mathrm{~N}-\mathrm{CH}_{3}\right), 4.14,4.27,5.15,5.84$ (4m, 5H, alditolyl H), 4.61(q, 2H, N-CH $\left.\mathrm{CH}_{3}\right)$, $7.29(\mathrm{~d}, 1 \mathrm{H}, \mathrm{N}=\mathrm{CH}), 11.88(\mathrm{~s}, 1 \mathrm{H}$, exchangeable, $=\mathrm{N}-\mathrm{NH}-)$; ${ }^{3} \mathrm{C} \mathrm{NMR}\left(75 \mathrm{MHz}, \mathrm{DMSO}-\mathrm{d}_{6}\right) \delta$ $15.8\left(\mathrm{C}-7 \mathrm{CH}_{3}\right), 20.3-20.9\left(4 \mathrm{CH}_{3}\right), 27.2\left(\mathrm{C}-7 \mathrm{CH}_{2}\right), 29.1(\mathrm{C}-3), 38.8$ (C-1), 62.0-72.1 (C-alditol), 101.8 (C-5), 144.5 (C-4), $147.1(\mathrm{C}-8), 149.0(\mathrm{~N}=\mathrm{CH}), 150.2$ (C-2), 152.5 (C-6); 169.2-171.9 (4 CO); MS: m/z (\%) = $539\left(\mathrm{M}+{ }^{+} 1,52\right), 537$ (27), 480 (2.0), 391 (5.0), 358 (2.0), 316 (3.0), 299 (3.0), 279 (3.0), 249 (5.0), 224 (7.0), 222 (100). Anal. calcd. for $\mathrm{C}_{22} \mathrm{H}_{30} \mathrm{~N}_{6} \mathrm{O}_{10}$ (538): C, 49.07; H, 5.62; N, 15.61. Found: C, 49.00; H, 5.45; N, 15.50\%.

2,3,4,5-Tetra- $O$-acetyl-D-arabinose-7-ethyl-8-theophyllinyl-hydrazone 16. Yield 30\%; yellow crystals, mp $154-157{ }^{\circ} \mathrm{C}$; IR (KBr) v $3232(\mathrm{NH}), 1745\left(\mathrm{COCH}_{3}\right), 1697,1653(\mathrm{C}=\mathrm{O}) \mathrm{cm}^{-1} ;{ }^{1} \mathrm{H}$ NMR (300 MHz, DMSO-d $) \delta 1.41\left(\mathrm{t}, 3 \mathrm{H}, \mathrm{N}-\mathrm{CH}_{2} \mathrm{CH}_{3}\right), 1.9-2.08\left(\mathrm{~m}, 12 \mathrm{H}, 4 \mathrm{COCH}_{3}\right), 3.24,3.37$ $\left(2 \mathrm{~s}, 6 \mathrm{H}, 2 \mathrm{~N}-\mathrm{CH}_{3}\right), 4.22,4.33,5.35,5.86$ (4m, 5H, alditolyl H), 4.64 (q, 2H, N-CH $\left.\mathrm{CH}_{3}\right), 7.29$ (d, $1 \mathrm{H}, \mathrm{N}=\mathrm{CH}), 11.95$ (s, 1H, exchangeable, $=\mathrm{N}-\mathrm{NH}-)$; ${ }^{3} \mathrm{C}$ NMR $\left(75 \mathrm{MHz}, \mathrm{DMSO}-\mathrm{d}_{6}\right) \delta 15.3(\mathrm{C}-7$ $\left.\mathrm{CH}_{3}\right), 20.4-20.9\left(4 \mathrm{CH}_{3}\right), 27.2\left(\mathrm{C}-7 \mathrm{CH}_{2}\right), 29.2$ (C-3), 38.5 (C-1), 61.5-72.0 (C-alditol), 101.9 (C5), $145.0(\mathrm{C}-4), 147.3(\mathrm{C}-8), 148.8(\mathrm{~N}=\mathrm{CH}), 150.8$ (C-2), 152.0 (C-6); 169.2-171.8 (4 CO); MS: $\mathrm{m} / \mathrm{z}(\%)=539\left(\mathrm{M}+1^{+}, 34\right), 525$ (13), 479 (3.0), 316 (3.0), 308 (23), 307 (13), 289 (8.0), 279 (4.0), 249 (6.0), 224 (8.0), 222 (100). Anal. calcd. for $\mathrm{C}_{22} \mathrm{H}_{30} \mathrm{~N}_{6} \mathrm{O}_{10}$ (538): C, 49.07; H, 5.62; N, 15.61. Found: C, 49.01; H, 5.28; N, 15.58\%.

\section{Reaction of products 7-11(or 12-16) with a mixture of bromine/ acetic acid/ sodium acetate}

To a suspension of 7-11 or 12-16 $(2.2 \mathrm{mmol})$ and anhydrous sodium acetate $(6.6 \mathrm{mmol})$ in acetic acid $(15 \mathrm{ml})$, a solution of bromine $(2.2 \mathrm{mmol})$ in acetic acid $(7 \mathrm{ml})$ was added dropwise and the mixture stirred at room temperature until reaction was judged to be complete by TLC. The solvent was evaporated under vacuum; the oily residue obtained was extracted with chloroform. The precipitate formed was collected, washed with ethanol and recrystallized from ethanol to give orange crystals of product, 8-bromo-7-ethyltheophylline $17^{37}$ (45\% yield), mp $170{ }^{\circ} \mathrm{C}$.

\section{Reaction of product 17 with hydrazine hydrate}

A mixture of $17(0.29 \mathrm{~g}, 1 \mathrm{mmol})$ in ethanol $(2 \mathrm{ml})$ and hydrazine hydrate $(0.2 \mathrm{ml}, 98 \%)$ was heated at $100{ }^{\circ} \mathrm{C}$ for $2 \mathrm{~h}$, then left to cool and poured into cold water. The solid product was filtered off, washed with cold water, dried and finally recrystallized from ethanol. The product 
obtained was identical in all respect to 7-ethyl-8-hydrazino-theophylline $\mathbf{1}(0.21 \mathrm{~g}, 90 \%$ yield $)$, mp 254-256 ${ }^{\circ} \mathrm{C}$ [lit. mp $\left.257-8{ }^{\circ} \mathrm{C}\right] .{ }^{28}$

\section{Antimicrobial evaluation}

Cultures of four fungi namely Aspergillus fumigatus AF, Penicillium italicum PI, Syncephalastrum racemosum $\boldsymbol{S} \boldsymbol{R}$ and Candida albicans $\boldsymbol{C A}$ were tested as well as four bacteria species namely Escherichia coli EC, Bacillus subtilis BS, Pseudomonas aeruginosa $\boldsymbol{P A}$ and Staphylococcus aureus $\boldsymbol{S} \boldsymbol{A}$ were used to investigate the antimicrobial activity of the compounds 9 and 12-16. The antimicrobial activity was assayed biologically using diffusion plate technique. The experiments were carried out by pouring a spore suspension of the fungal species (one ml. of sterile water contains approximately $10^{8}$ conidia) or spreading bacterial suspension over a solidified malt agar medium. The layer was allowed to set for $30 \mathrm{~min}$. A solution of the test compounds 9 and 12-16 $(5.0,2.5,1.0 \mathrm{mg} / \mathrm{ml})$ was placed onto sterile $5 \mathrm{~mm}$ filter paper discs and allowed to dry, then the discs were placed on the center of the malt agar plate and incubated at optimum incubation temperature $28 \pm 2{ }^{\circ} \mathrm{C}$. Test organism growth may be affected by the inhibitory action of the test compound. So, a clear zone around the disc appeared as an indication of the inhibition of the test organism growth. The size of clearing zone is proportional to the inhibitory action of the test compound. The fungicide Terbinafin and the bactericide Chloramphenicol were used as standards under the same conditions. Measurements were considered after $72 \mathrm{~h}$ for fungi and $24 \mathrm{~h}$ for bacteria. The results are shown in Table 1.

\section{References}

1. Corsano, S.; Scapiochi, R.; Strappaghetii, G. Arch. Pharm. (Weinheim) 1994, 327, 411.

2. Baziard-Mouysset, G.; Rached, A.; Younes, S.; Tournaire, C.; Stigliani, J. L.; Payard, M.; Yavo, J. C.; Advenier, C. Eur. J. Med. Chem. 1995, 30, 253.

3. Sandoz, A. G. Israeli IL 1992, 83, 659; Chem. Abstr. 1992, 117, 7957k.

4. Gajewczyk, L.; Zejc, A. Acta. Pol. Pharm. 1992, 49, 61.

5. Parrick, J.; Mehta, L. K. J. Heterocycl. Chem. 1993, 30, 323.

6. Bovy, P. R.; Collins, J. T.; Chamberlain, T. S.; Gheng, P. K. PCT Int. Appl. WO 9207852 , Chem. Abstr. 1992, 117, 131218j.

7. Kuzmenko, V. V.; Pozharskii, A. F.; Chemyshev, A. J.; Nanavyan, I. M. Khim. Geterotsikl. Soedin. 1987, 11, 1551, Chem. Heterocycl. Comp. 1988, 240, 94.

8. Hesek, D.; Tegza, M.; Rybar, A.; Povazanec, F. Synthesis 1989, 681.

9. Dreier A.; Haller, R. Arch. Pharm. (Weinheim) 1987, 320, 1004.

10. Nanavyan, I. M.; Kuzmenko, V. V.; Pozharskii, A. F.; Klyuev, A. N. Khim. Geterotsikl. Soedin. 1987, 10, 1398; Chem. Heterocycl. Comp. 1988, 206, 97.

11. Karczmarzyk, Z.; Karolak-Wojciechowska, J.; Pawlowski, M. Acta. Cryst. 1991, 47, 1902.

12. Karolak-Wojciechowska, J.; Pawlowski, M. J. Crystallogr. Spectrosc. Res. 1990, $20,477$. 
13. Pawlowski, M. Pharmazie 1987, 42, 371.

14. Pawlowski, M.; Buschauer, A.; Schunack, W. Arch. Pharm. (Weinheim) 1989, 322, 447.

15. Jin, R. H; Nisshjkubo, T. Tetrahedron Lett. 1992, 33, 6307.

16. Blythin, D. J.; Kamiinski, J. J.; Domalski, M. S.; Spitler, J.; Solomon, D. M.; Conn, D. J.; Shing-Chun, W.; Lehman, L. V.; Bober, L. A.; Chiu, P. J. S.; Watnick, A. S.; Siegel, M. I.; Hibert, J. M.; McPhail, A. T. J. Med. Chem. 1986, 29, 1099.

17. Conn, D. J.; Kamiinski, J. J.; Solomon, D. M.; Mc Phail, A. T. J. Org. Chem. 1988, 53, 3265.

18. D. M. Solomon, D. M.; Kamiinski, J. J. US Pat 4,816,458; Chem. Abstr. 1989, 111, 153833j.

19. El Ashry, E. S. H.; Y.; El Kilany, Y. Adv. Heterocycl. Chem. 1997, 67, 391.

20. El Ashry, E. S. H.; El Kilany, Y. Adv. Heterocycl. Chem. 1997, 68, 1.

21. El Ashry, E. S. H.; El Kilany, Y. Adv. Heterocycl. Chem. 1998, 69, 129.

22. Shaban, M. A. E.; Nasr, A. Z. Adv. Heterocycl. Chem. 1997, 68, 223.

23. Shaban, M. A. E. Adv. Heterocycl. Chem. 1998, 70, 163.

24. (a) Klosa, J. J. Prakt. Chem. 1958, 6, 187. (b) Shaban, M. A.; Taha, M. A. M.; Morgaan, A. E. A. Monatsh. Chem. 2000, 131, 487.

25. Mosselhi, M. A. N. Nucleos. Nucleot. Nucl. 1993, 12, 431.

26. Mosselhi, M. A. N. Nucleos. Nucleot. Nucl. 1999, 18, 2043.

27. Mosselhi, M. A. N. Seliger, H. J.Chem. Res. (S) 2001, 359.

28. Damiens, R.; Delaby R. Bull. Soc. Chim. Fr. 1955, 888.

29. Mosselhi, M. A. N.; Abbass, I. M. Bull. Pol. Acad. Sci. 1993, 41, 179.

30. Rashed, N.; Ibrahim, E. I.; El-Ashry, E. S. H. Carbohydrate Res. 1994, 254, 295.

31. Shawali, A. S.; Hassaneen, H. M.; Shurrab, N. K. Tetrahedron 2008, 64, 10339.

32. Ciesielski, M.; Pufky, D.; Doering, M. Tetrahedron 2005, 61, 5942.

33. Shaban, M. A., Taha, M. A. M.; Nasr, A. Z.; Morgaan, A. E. A. Pharmazie 1995, 50, 784.

34. Shaban, M. A.; Taha, M. A. M.; Nasr, A. Z. Pharmazie 1996, 51, 707.

35. Taha, M. A. M.; El-Badry, S. M. J. Chin. Chem. Soc. 2006, 53, 1181.

36. Mosselhi, M. A. N.; Tawfik, N. M.; Shawali, A. S. Monatsh. Chem. 2003, 134, 565.

37. Aweiler-Smialek, J.; Loson, W. Med. Acad. Cracow, Pol. Dissertationes Pharmaceuticae et Pharmacologicae 1969, 21, 367.

38. Mosselhi, M. A.; Pfleiderer, W. J. Heterocycl. Chem. 1993, 30, 1221. 